
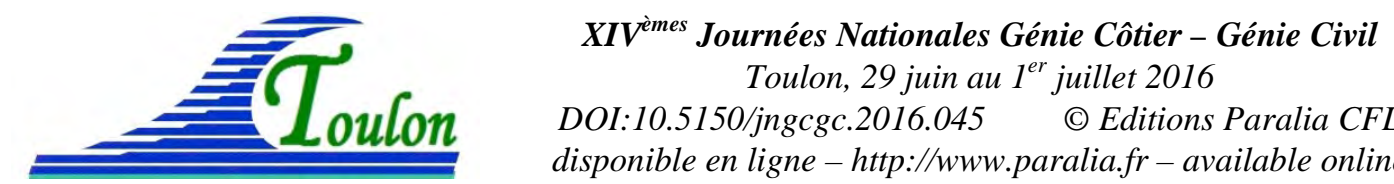

DOI:10.5150/jngcgc.2016.045 C Editions Paralia CFL

disponible en ligne - http://www.paralia.fr - available online

\title{
Restitution bathymétrique à partir de la mesure de la célérité et de la longueur d'onde des vagues par imagerie satellitaire optique
}

\author{
Adrien POUPARDIN ${ }^{1,2}$, Déborah IDIER ${ }^{1}$, \\ Marcello DE MICHELE ${ }^{1}$, Daniel RAUCOULES ${ }^{1}$
}

1. BRGM, 3 avenue Claude Guillemin, F-45060 Orléans Cedex 2, France.

2. Actuellement au CEA DAM DIF, F-91297 Arpajon Cedex, France.

adrien.poupardin@cea.fr

\section{Résumé :}

La méthode présentée permet d'estimer la bathymétrie de zones littorales à partir d'un seul jeu de données du satellite SPOT-5, en s'appuyant sur l'estimation des caractéristiques de vagues (longueurs d’onde et célérité) et de la théorie linéaire des vagues (relation de dispersion). La méthode des ondelettes est utilisée pour extraire les ondes dominantes, leurs longueurs d'onde et leurs directions. Puis, leur célérité est estimée à partir du décalage temporel (2,04 s) existant entre les images panchromatique et multi-spectrale du satellite SPOT-5. A partir de ces caractéristiques, la profondeur d'eau est estimée en utilisant la relation de dispersion. La méthode dénommée CWB (Correlation, Wavelet et Bathymetry) a été testée sur la zone de Saint-Pierre à La Réunion. La bathymétrie a été estimée sur une grille de $80 \mathrm{~m} \times 80 \mathrm{~m}$ et les résultats ont été comparés à des mesures de bathymétrie in-situ (LIDAR et sondeurs multifaisceaux). L'erreur relative moyenne est inférieure à 30\% sur la gamme de profondeurs 3-80 m.

Mots clés : Vagues, Célérité, Longueur d’onde, Ondelettes, Imagerie satellitaire optique.

\section{Introduction}

Aujourd'hui, la bathymétrie littorale est une donnée indispensable à toute étude d'aléa ou risque de submersion, ou tout simplement pour caractériser la morphologie des fonds marins. L'estimation de la bathymétrie est envisageable à partir de données satellitaires radars ou optiques et permet d'alléger considérablement les coûts et les délais de ce type d'études.

Parmi les études d'estimation de la bathymétrie existantes, l'imagerie satellitaire multispectrale permet de mesurer la bathymétrie en utilisant les propriétés optiques des eaux en petits fonds (CAPO et al., 2013). Néanmoins, cette technique s'applique principalement à proximité immédiate de la côte, pour les très faibles profondeurs d'eau. Par exemple, CAPO et al. (2013) estiment la bathymétrie jusqu'à des profondeurs d'environ $4 \mathrm{~m}$.

D’autres techniques satellitaires, radar et optique, utilisent les caractéristiques de vague mesurables pour remonter à une estimation de la bathymétrie. En effet, la plupart de ces 
techniques sont basées sur la relation de dispersion issue de la théorie linéaire des vagues :

$h=\frac{\lambda}{2 \pi} \cdot \tanh ^{-1}\left(\frac{2 \pi c^{2}}{g \lambda}\right)$

avec $h$ la hauteur d'eau, $c$ la célérité (vitesse de phase), $\lambda$ la longueur d'onde des vagues, et $g$ l'accélération de la pesanteur. La relation de dispersion est particulièrement bien adaptée pour déterminer la bathymétrie en profondeur intermédiaire (c.a.d. telle que $\lambda / 20<h<\lambda / 2$ ). Au niveau de la zone de surf, le déferlement des vagues et les effets non linéaires augmentent considérablement l'erreur sur son estimation (HOLLAND, 2001). La hauteur de vague $H$ doit alors être prise en compte pour améliorer l'estimation de la profondeur d'eau $h$ (STOCKDON \& HOLMAN, 2000 ; CATALAN \& HALLER, 2008).

L'utilisation de la relation de dispersion pour estimer la bathymétrie repose sur le choix des couples $(c, \lambda)$ ou $(f, \lambda)$ avec $f$ la fréquence de houle, ces grandeurs variant avec la bathymétrie dans le cas de houles réelles. La plupart des techniques satellitaires aussi bien optique (LEU \& CHANG, 2005) que radar (PLESKACHEVSKY \& LEHNER, 2011) utilisent la fréquence $f$ de la houle déterminée au large et la considère constante lorsque la houle se rapproche de la côte. La fréquence de houle peut également être mesurée sur le terrain par l'intermédiaire de bouées (MARIEU et al., 2012) ce qui permet de pouvoir déterminer localement l'évolution temporelle de la houle.

L'étude de LEU et CHANG (2005) considère que la houle est une onde monochromatique ce qui n'est pas toujours le cas en pratique. PLESKACHEVSKY et LEHNER (2011) vont plus loin en utilisant une technique qui permet de localiser l'onde la plus énergétique au large et de la suivre lorsqu'elle se propage vers la côte. La technique radar utilisée (radar à synthèse d'ouverture -SAR) permet de caractériser les ondes longues (houle) car celles-ci modulent les ondes plus courtes (rides) qui sont visibles par SAR. Néanmoins, en dessous d'une valeur de longueur d'onde seuil, le processus de reconstruction de l'image radar à partir de SAR n'est plus possible.

Les travaux préliminaires de DE MICHELE et al. (2012) ont permis de montrer qu'il est possible de déterminer la célérité de la houle de manière directe à partir d'imagerie satellitaire optique issue du capteur SPOT-5. Ceci représente une opportunité pour déterminer localement plusieurs couples $(c, \lambda)$ afin d'estimer la bathymétrie en utilisant la relation de dispersion.

Ces résultats ont mené à explorer l'utilisation des images SPOT-5 pour estimer la bathymétrie en zone côtière, avec pour objectif principal de pouvoir accéder à des informations bathymétriques là où aucune mesure in-situ n'est disponible (voir POUPARDIN et al., 2014 et POUPARDIN et al., 2015). 


\section{XIV èmes Journées Nationales Génie Côtier - Génie Civil \\ Toulon, 29 juin au $1^{\text {er }}$ juillet 2016}

\section{La méthode CWB (for Celerity, Wavelet et Bathymétrie)}

La méthode développée pour estimer la bathymétrie s'appuie sur : (i) l'adaptation de la méthode d'estimation de la célérité de DE MICHELE et al. (2012) pour associer une célérité $c$ à chaque longueur d'onde dominante $\lambda$; (ii) l'estimation de la bathymétrie à partir de ces couples $(c, \lambda)$.

Les caractéristiques de vagues sont extraites des images panchromatique et multispectrale du satellite SPOT-5 en y appliquant une analyse en ondelettes. Ces caractéristiques sont ensuite utilisées pour déterminer la bathymétrie à partir de la relation de dispersion (1).

Ainsi, la méthodologie pour estimer la bathymétrie est détaillée en cinq étapes (figure 1) :

- (1) détermination du spectre des vagues en différents points d'une grille de $20 \mathrm{~m} \times$ $20 \mathrm{~m}$ en utilisant une analyse en ondelettes ;

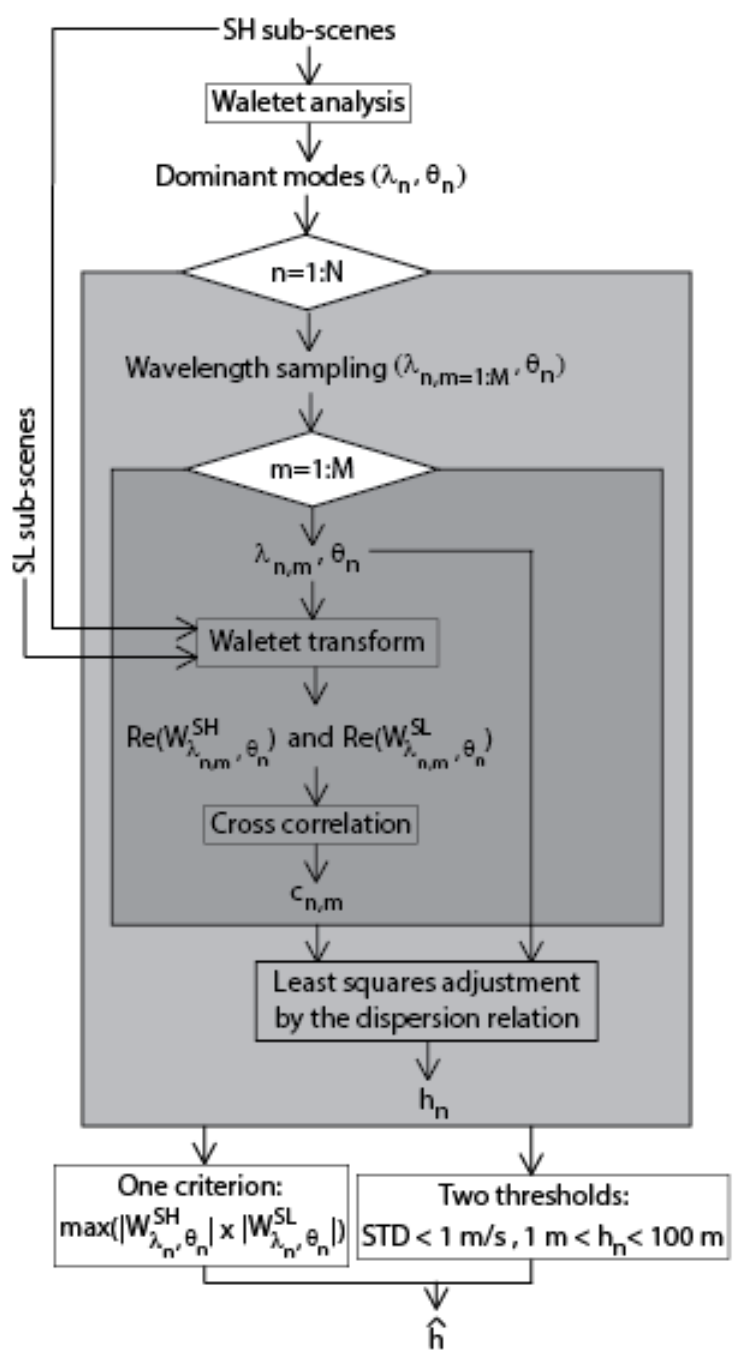

Figure 1. Description de la méthodologie (Tiré de POUPARDIN et al., 2015). 
- (2) extraction de ondes dominantes caractérisées par leur longueur d'onde et par leurs directions

- (3) pour chaque onde dominante , utilisation des images (panchromatique et multispectrale) et du décalage temporel entre ces deux images pour calculer célérités correspondant à la gamme de longueurs d’ondes centrée sur la longueur d'onde dominante , en conservant toujours la même orientation ;

- (4) utilisation du nuage de points dans le plan pour déterminer la profondeur d'eau qui minimise la somme des écarts au carré entre le nuage de points et la courbe de dispersion (algorithme de Levenberg-Marquardt) ;

- (5) choix de la profondeur d'eau parmi les ondes dominantes.

\section{Description du site d'étude et des données utilisées}

La méthode a été testée en utilisant les images du satellite SPOT-5 prises le 6 Février 2010 à 10h30 (1h30 avant la marée basse) dans la zone de Saint-Pierre au sud-ouest de l'île de La Réunion (voir figure 2).

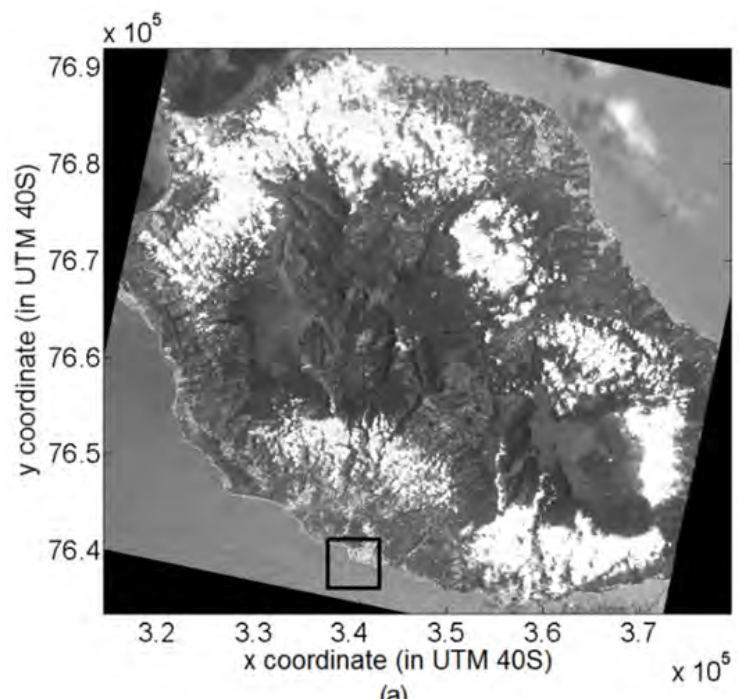

(a)

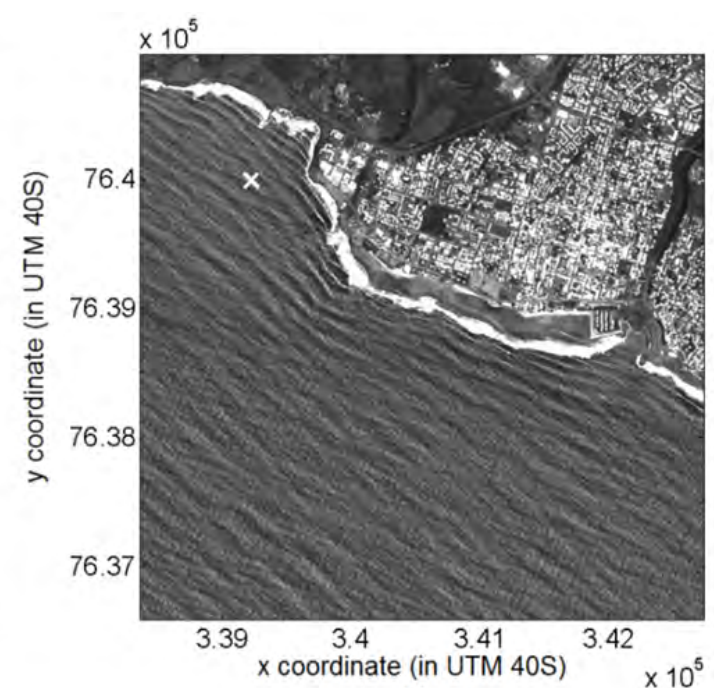

(b)

Figure 2. Image panchromatique de SPOT-5: (a) île de La Réunion; (b) zoom sur la zone de Saint-Pierre (Tiré de POUPARDIN et al., 2015).

La zone de Saint-Pierre est caractérisée par un plateau continental étroit où les données existantes montrent un courant négligeable à cette date (entre 0.1 et $0.14 \mathrm{~m} / \mathrm{s}$ (Navy Coastal Ocean Model of the National Oceanic and Atmospheric Administration)). Ce site a été choisi car des données haute résolution y sont disponibles, par LIDAR (Produits Litto3D, voir www.shom.fr pour plus de détails) pour les profondeurs inférieures à $30 \mathrm{~m}$ et par sondeur multi-faisceaux pour les plus grandes profondeurs (SHOM, Service Hydrographique et Océanographique de la Marine). 


\section{XIV èmes Journées Nationales Génie Côtier - Génie Civil \\ Toulon, 29 juin au $1^{\text {er }}$ juillet 2016}

Le satellite SPOT-5 est équipé de capteurs haute résolution (HRG1-2) qui lui permettent d'acquérir une image multispectrale (XS1-3) et une image panchromatique (HMA) à des résolutions de 10 et $2.5 \mathrm{~m}$ respectivement. Le décalage temporel entre ces images est de $D T=2,04 \mathrm{~s}$. Cette configuration permet de suivre un ensemble de pixels dans leur déplacement (DE MICHELE et al., 2012). C’est la bande verte (XS1) de l'image multispectrale qui a été utilisée pour la correspondance avec l'image panchromatique (HMA) car sa largeur de bande est centrée sur $0.55 \mu \mathrm{m}$ se rapprochant ainsi au mieux de celle de l'image panchromatique.

En outre, les images doivent répondre à un certain nombre de conditions pour que la méthode soit applicable. La couverture nuageuse sur la mer doit être quasiment nulle. Les positions relatives du soleil et du satellite par rapport à la zone d'étude ont également un impact important sur la qualité des images. En outre, la cambrure des vagues (c.a.d leur amplitude sur leur longueur d'onde) doit être suffisante pour que les variations de réflectance de la lumière soient significatives et que l'image des vagues affiche le meilleur contraste possible. Par ailleurs, nous recommandons certaines conditions indispensables en termes de longueur d'onde et de célérité des vagues. Ainsi, la résolution de l'image multispectrale (ici l'image XS1 avec une résolution de $10 \mathrm{~m}$ ) doit être suffisamment fine par rapport à la longueur d'onde des vagues afin de se placer dans les conditions d'applicabilité du théorème de Nyquist. L'écart temporel,DT, entre les deux images doit être suffisamment grand, ou la résolution de l'image panchromatique (ici l'image HMA de résolution $2.5 \mathrm{~m}$ ) suffisamment bonne, pour permettre aux vagues de se déplacer de plusieurs pixels. Enfin, DT ne doit pas être trop long afin de capter au mieux le déplacement des vagues qui peuvent être impactées par de la réfraction et dont la vitesse décroit rapidement en phase de shoaling.

\section{Résultats}

La bathymétrie estimée par la méthode CWT a été obtenue à une résolution spatiale de $20 \mathrm{~m} \times 20 \mathrm{~m}$. Néanmoins, la résolution réelle de la bathymétrie ne peut pas être aussi fine puisque la taille des sous-images utilisées pour l'inter-corrélation est de $320 \mathrm{~m} \times$ $320 \mathrm{~m}$ offshore et de $160 \mathrm{~m} \times 160 \mathrm{~m}$ près des côtes, ces sous-images se recouvrant les unes, les autres. La bathymétrie estimée par la méthode CWT est donc interpolée sur une grille de $80 \mathrm{~m} \times 80 \mathrm{~m}$ (figure 3 (b)).

Les résultats de bathymétrie ont été comparés aux mesures in-situ également interpolées sur la grille de $80 \mathrm{~m} \times 80 \mathrm{~m}$ (figure 3 (a)). Pour illustrer la qualité de la méthode, la figure 4 montre la bathymétrie estimée $\widehat{h}$ en fonction des mesures de bathymétrie in-situ $h_{\text {in-situ }}$. 


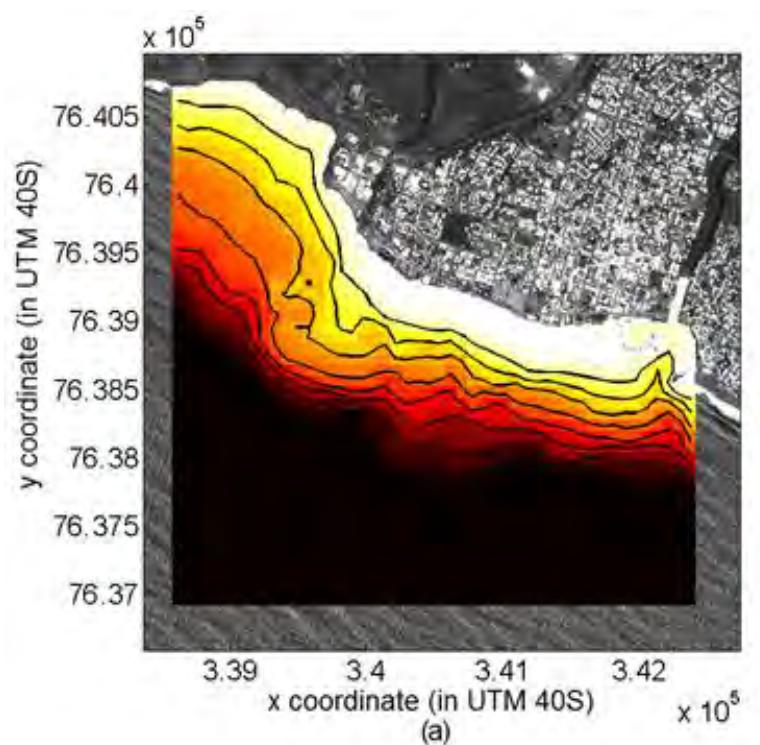

(a)

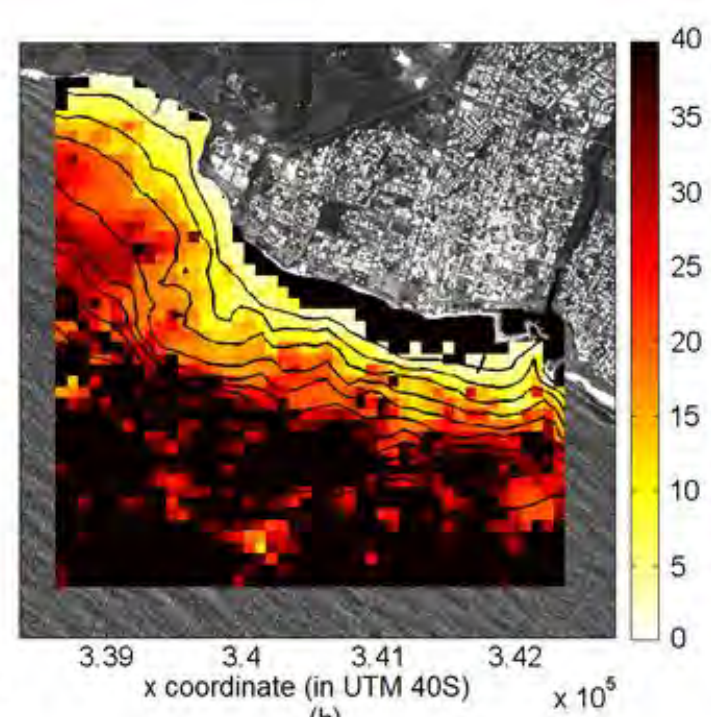

(b)

Figure 3. Bathymétries (a) mesurée, (b) estimée par la méthode CWT (in m) sur la zone de Saint-Pierre sur l'île de La Réunion - Résolution de la grille : $80 \mathrm{~m} \times 80 \mathrm{~m}$ Isobathes représentées en noir de 0 à $50 \mathrm{~m}$ de profondeur avec un pas de $5 \mathrm{~m}$ (Tiré de POUPARDIN et al., 2015).

Sur la figure 4, la dispersion peut être considérée comme faible jusqu’à $h_{\text {in-situ }}=$ $25 \mathrm{~m}$. Puis, elle augmente régulièrement pour les profondeurs plus importantes.

De plus, les centiles (croix bleues sur la figure 4) représentés à partir de $h_{\text {in-situ }}=2 \mathrm{~m}$ montrent qu'en moyenne la méthode est fiable sur la gamme de profondeur in-situ [2 m, $25 \mathrm{~m}$ ]. Une dérive apparaît à partir de $h_{\text {in-situ }}=25 \mathrm{~m}$. Les résultats au-delà de ce seuil doivent tout de même être considérés même s'ils ne sont pas d'aussi bonne qualité que des mesures in-situ. Cela permet d'estimer une bathymétrie dans les zones où il n'y a pas d'autre information disponible.

La figure 5 montre les erreurs absolues et relatives moyennes par gamme de profondeur (moyennes appliquées aux valeurs absolues des erreurs): [1 m, $2 \mathrm{~m}],[2 \mathrm{~m}, 3 \mathrm{~m}]$, [3 m, $5 \mathrm{~m}],[5 \mathrm{~m}, 10 \mathrm{~m}],[10 \mathrm{~m}, 15 \mathrm{~m}], \quad[15 \mathrm{~m}, 20 \mathrm{~m}], \quad[25 \mathrm{~m}, 30 \mathrm{~m}], \quad[30 \mathrm{~m}, 35 \mathrm{~m}]$, [35 m, $40 \mathrm{~m}],[40 \mathrm{~m}, 50 \mathrm{~m}],[50 \mathrm{~m}, 60 \mathrm{~m}],[60 \mathrm{~m}, 70 \mathrm{~m}]$ and $[70 \mathrm{~m}, 90 \mathrm{~m}]$. 


\section{XIV èmes Journées Nationales Génie Côtier - Génie Civil \\ Toulon, 29 juin au $1^{\text {er }}$ juillet 2016}

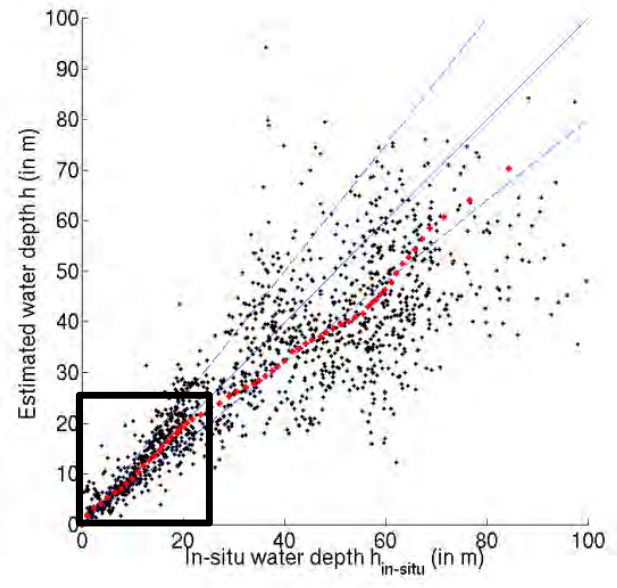

(a)

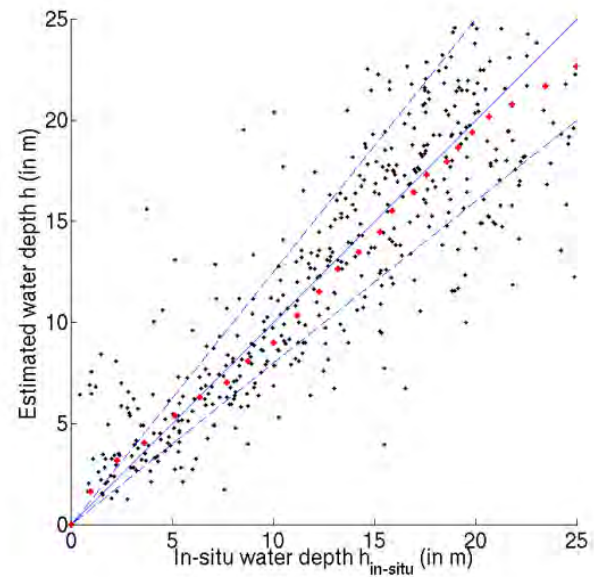

(b)

Figure 4. Profondeur d'eau estimée $\hat{h}$ en fonction des mesures de bathymétrie in-situ $h_{\text {in-situ }}$ en considérant tous les points de la grille de $80 m \times 80 m$-Les croix rouges représentent les centiles en partant de $h_{\text {in-situ }}=2$ (Tiré de POUPARDIN et al., 2015).

A partir de la figure 5, on constate notamment que l'erreur relative moyenne est inférieur à $24 \%$ dans la gamme de profondeur [ $5 \mathrm{~m}, 35 \mathrm{~m}$ ] et inférieur à $28 \%$ dans la gamme $[35 \mathrm{~m}, 90 \mathrm{~m}]$. Il faut préciser ici, que seules les erreurs moyennes sont présentées et que localement les erreurs absolue et relative peuvent se rapprocher de 0 . Par exemple, la zone à l'ouest de la carte (figure 2 (a)) présente des pentes plus douces (autour de $2 \%$ ) qui induisent une réduction de l'erreur commise par la méthode. Dans les zones où les pentes sont plus fortes (supérieures à 5 \%), à l'est de la carte, au niveau du port de Saint-Pierre, la méthode peut également être appliquée bien qu'elle induise des erreurs légèrement plus fortes. L'erreur commise par la méthode peut également être induite par un manque d'informations sur certaines zones de l'image où les vagues ne sont pas suffisamment bien décrites par les écarts de réflectance lumineuse.

Ces résultats sont satisfaisants au regard de la littérature existante, en considérant que les valeurs de bathymétrie sont exprimées sur une grille de $80 \mathrm{~m} \times 80 \mathrm{~m}$. En effet, LEU et CHANG (2005) obtiennent une erreur relative de seulement $16.2 \%$ sur la gamme de profondeur [ $2 \mathrm{~m}, 20 \mathrm{~m}$ ], mais leur résolution est de $200 \mathrm{~m} \times 200 \mathrm{~m}$ et la bathymétrie est caractérisée par une pente 2 ou 3 fois plus douce que dans notre étude. Des études de sensibilité ainsi que des recommandations pour l'utilisation de la méthode sont détaillées dans POUPARDIN et al. (2015). 


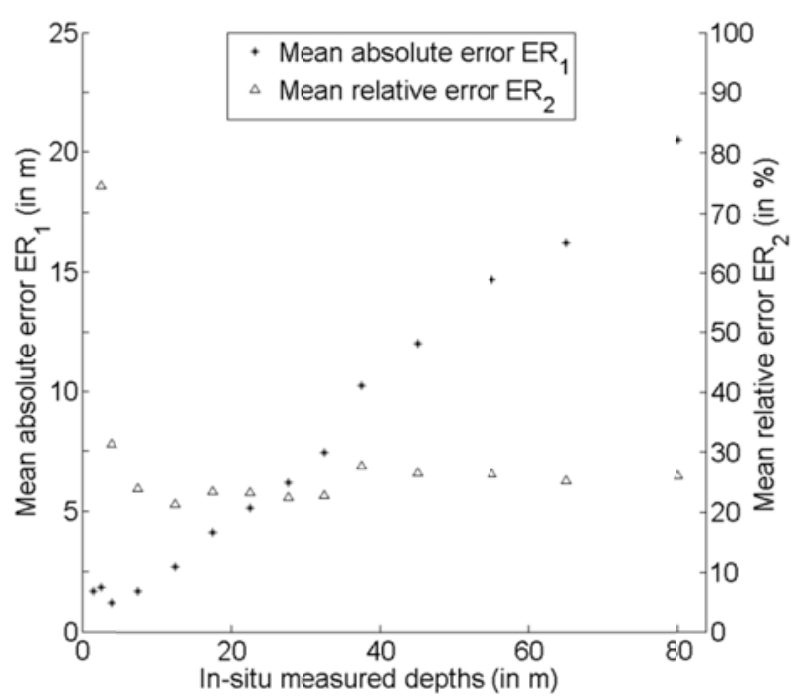

Figure 5. Moyenne de la valeur absolue des erreurs absolues (*) et relatives $(\Delta)$ sur la bathymétrie (respectivement en $m$ et en \%) pour plusieurs gammes de profondeurs :

[1 $\mathrm{m}, 2 \mathrm{~m}],[2 \mathrm{~m}, 3 \mathrm{~m}],[3 \mathrm{~m}, 5 \mathrm{~m}],[5 \mathrm{~m}, 10 \mathrm{~m}],[10 \mathrm{~m}, 15 \mathrm{~m}],[15 \mathrm{~m}, 20 \mathrm{~m}]$, [25 m, 30 m], [30 m, 35 m], [35 m, $40 \mathrm{~m}],[40 \mathrm{~m}, 50 \mathrm{~m}],[50 \mathrm{~m}, 60 \mathrm{~m}],[60 \mathrm{~m}, 70 \mathrm{~m}]$ et [70 m,90 m] (Tiré de POUPARDIN et al., 2015).

\section{Conclusion}

La méthode CWT utilise une analyse en ondelettes couplée avec un calcul d'intercorrelation afin de déterminer $N$ nuages de points dans le plan $(\lambda, c)$, correspondant à $N$ ondes dominantes. La relation de dispersion est ensuite utilisée pour déterminer les profondeurs d'eau associées à ces ondes. Enfin, la "meilleure" profondeur d'eau estimée est sélectionnée à partir d'une analyse sur les spectres d'énergie.

Cette méthode a permis de retrouver la bathymétrie à partir d’imagerie satellitaire optique à une précision jugée acceptable pour l’objectif visé : 20 à 30\% d'erreur pour la gamme de profondeur $3-80 \mathrm{~m}$, avec certaines zones où l'erreur est localement proche de $0 \%$.

Néanmoins, la méthode peut encore être améliorée et représente une réelle opportunité pour estimer la bathymétrie là où aucune autre méthode plus classique ne pourrait être appliquée, ou bien pour estimer une bathymétrie dans le passé sous réserve de disposer des images satellitaires archivées avec une résolution suffisante (par exemple avant un événement, type glissement de terrain ou cyclone). Il serait notamment intéressant de considérer un calcul sub-pixellique de la célérité des vagues afin d'améliorer cette caractéristique de vague qui impacte fortement le calcul de la bathymétrie. De même, l'utilisation d'une paire d'images satellitaire avec une meilleure résolution (telles que les images Pléiades) permettrait de mieux capter les rides de vagues se propageant sur les ondes les plus longues. Ceci permettrait notamment de pouvoir considérer les 


\section{XIV ${ }^{\text {èmes }}$ Journées Nationales Génie Côtier - Génie Civil \\ Toulon, 29 juin au $1^{\text {er }}$ juillet 2016}

courants dans la relation de dispersion. En effet, ces derniers, s'ils sont importants, peuvent générer une erreur non négligeable sur le calcul de la bathymétrie.

A terme, la méthode pourra être généralisée en considérant, non pas une paire d'images, mais une multitude d'images satellitaire optiques permettant de calculer les spectres spatio-temporels associés à la houle. L'analyse de ces spectres pourra conduire à une estimation plus précise de la bathymétrie et des courants, de la même manière que dans la méthode de PIOTROWSKI et DUGAN (2002).

\section{Remerciements}

Nous souhaitons remercier l'institut Carnot BRGM et le programme national de télédétection spatiale (PNTS) pour leur financement.

Nous remercions également l'équipe Kalideos (CNES) pour les données SPOT-5, ainsi que le SHOM (Service Hydrographique et Océanographique de la Marine) et l'IGN (Institut national de l'information géographique et forestière) pour les données de bathymétrie.

\section{Références bibliographiques}

CAPO S., MARIEU V., BRU D., LUBAC B., BONNETON P. (2013). Decadal morphodynamics evolution of a mixed-energy inlet using multispectral SPOT imagery. Coastal Dynamics, pp 283-294.

CATALAN P., HALLER M. (2008). Remote sensing of breaking wave phase speeds with application to nonlinear depth inversion. Coastal Engineering, Vol. 55, pp 93-111. http://dx.doi.org/10.1016/j.coastaleng.2007.09.010

HOLLAND T.K. (2001). Application of the linear dispersion relation with respect to depth inversion and remotely sensed imagery. IEEE Transactions on Geoscience and Remote Sensing, Vol. 39(11), pp 2060-2071. http://dx.doi.org/10.1109/36.951097

DE MICHELE M., LEPRINCE S., THIEBOT J., RAUCOULES D., BINET R. (2012). Direct measurement of ocean waves velocity field from a single SPOT-5 dataset. Remote Sensing of Environment, Vol. 119, pp 266-271. http://dx.doi.org/10.1016/j.rse.2011.12.014

LEU L., CHANG H. (2005). Remotely sensing in detecting the water depths and bed load of shallow waters and their changes. Ocean Engineering, Vol. 32, pp 1174-1198. http://dx.doi.org/10.1016/j.oceaneng.2004.12.005

MARIEU V., GUERIN T., CAPO S., BRU D., LUBAC B., HANQUIEZ V., LAFON V., BONNETON P. (2012). Bathymétrie de l'embouchure du bassin d'Arcachon par fusion de données hétéroclites et reconstruction bathymétrique. XII ${ }^{\mathrm{èmes}}$ JNGCGC, Cherbourg, pp 603-610. http://dx.doi.org/10.5150/jngcgc.2012.065-M

PIOTROWSKI C.C., DUGAN J. (2002). Accuracy of bathymetry and current retrievals from airborne optical time-series imaging of shoaling waves. In IEEE Transactions on 
Thème 3 - Instrumentation, mesures, imagerie et télédétection

Geosciences and Remote Sensing, Vol. 40(12), pp 2606-2618. http://dx.doi.org/10.1109/TGRS.2002.807578

PLESKACHEVSKY A., LEHNER S. (2011). Estimation of Underwater Topography using Satellite High Resolution Synthetic Aperture Radar Data. In Proc. of 4e TerraSAR-X Meeting, Oberpfaffenhofen, Germany, 14-16 Feb. 2011, pp 1-19.

POUPARDIN A., DE MICHELE M., RAUCOULES D., IDIER D. (2014). Water depth inversion from satellite dataset. In IEEE International Geosciences and Remote Sensing Symposium, Québec City, Canada, July 13-18. http://dx.doi.org/10.1109/igarss.2014.6946924 POUPARDIN A., IDIER D., DE MICHELE M., RAUCOULES D. (2016). Water depth inversion from a single SPOT-5 dataset. IEEE Transactions on Geosciences and Remote Sensing, Vol. 54, No. 4, pp 2329-2342. http://dx.doi.org/10.1109/TGRS.2015.2499379 STOCKDON H.F., HOLMAN R.A. (2000). Estimation of wave phase speed and nearshore bathymetry from video imagery. Journal of Geophysical Research, Vol. 105, pp 22 015-22 034. http://dx.doi.org/10.1029/1999jc000124 